\title{
Mathematical modeling of incentive mechanisms in projects for the development of new production
}

\author{
O.V. Pavlov ${ }^{1}$ \\ ${ }^{1}$ Samara National Research University, 34 Moskovskoe Shosse, 443086, Samara, Russia
}

\begin{abstract}
The incentive problem of executors of the new products development project at the industrial enterprise is considered in this article. Mastering of a new product leads to the learning effect, which implies reduction of time spent on performing repetitive tasks by workers, resulting in a dynamic change in the economic performance of production. The project of the new products development is considered as a managed hierarchical dynamic system, consisting of a project management board (principal) and teams of agents. The interaction of project participants is formalized as a hierarchical dynamic game. To solve the problem, a numerical algorithm is developed based on a sequential solution of two optimal control problems that are solved using the Bellman dynamic programming method.
\end{abstract}

Keywords: new products development project; learning effect; hierarchical dynamic game

\section{Introduction}

In the new products development project at industrial enterprises, employees have to master new types of work and equipment, which is associated with the acquisition of new professional skills. In the process of mastering, the learning effect manifests itself, which is that the time spent by workers on performing repetitive tasks is reduced [1, 2]. The learning effect leads to a dynamic change in the economic performance of production: the volume of output per unit of time, labor intensity and production costs.

Every time the cumulative volume of production doubles, the productivity of workers increases by 10-15 percent [1]. The cumulative volume of production means the number of products manufactured from the beginning of production as a cumulative result.

The new production development project at an industrial enterprise is regarded as a managed hierarchical dynamic system consisting of the manager of the project (principal) and executors (agents). The state of the hierarchical dynamic system in each period of time depends on its state and the actions of the participants in the previous period.

Production activity in the project of the new production development is characterized by the mismatching interests of the principal and agents, which leads to a decrease in economic efficiency. The solution of these contradictions is possible with coordinated management mechanisms that encourage agents to choose actions that are beneficial to the principal.

Dynamic models of interaction of unequal players are considered in the theory of Stackelberg's dynamic games [3] and the theory of dynamic games of Germeyer [4]. Applied models of the dynamic games theory in the field of economics and management are given in $[5,6]$.

In this dynamic game model there are dynamics of decision making and dynamics of the managed system. The inequality of participants is fixed by the moves order. The first move is made by the principal, who chooses his own strategy - the unit payment rate, - and reports it to the agents. The principal, knowing the agent's goal functions, maximizes his goal function, taking into account the optimal responses of agents. It is assumed that agents are not linked to each other and perform independently. Dynamic game model is considered in a discrete form, which reflects the nature of production activity.

\section{Statement of the dynamic problem of proportional incentive}

\subsection{The decision-making model of the principal}

A two-level dynamic production system consisting of the principal and $n$ independent agents is considered. Agents produce parts, from which the final product is assembled. Labor costs and financial incentives for agents depend only on their own actions.

The dynamics of the new product production is described by a discrete equation:

$$
x_{t}=x_{t-1}+u_{t}, \quad t=1, T,
$$

where $x_{t}$ - cumulative production volume of a new product for the $t$-th time period, $t$ - number of the time period, $u_{t}$ production volume of new product in the period $t, T$ - amount of time periods.

In the initial period, the number of products produced is as follows:

$$
x_{0}=X_{0} \text {, }
$$

In the final period, the cumulative volume of finished products should be equal to the specified volume:

$$
x_{T}=X_{0}+R \text {, }
$$

where $R$-specified number of finished products.

The production volume of products in each period $t$ is imposed by the following restrictions:

$$
Q^{\min } \leq u_{t} \leq Q^{\max }, t=1, T,
$$

$3^{\text {rd }}$ International conference "Information Technology and Nanotechnology 2017" 
where $Q_{i}^{\min }-$ minimum production volume, taking into account technological and logistic requirements, $Q^{\max }-$ maximum production volume limited by the production capacity of equipment.

The labor costs of manufacturing the product in period $t$ are defined as the multiplication of the product labour intensity $c_{p t}$ and production volume in this period $u_{t}$ :

$$
C_{p t}=c_{p t} u_{t}, t=1, T .
$$

Due to the learning effect, the product labor intensity decreases depending on the cumulative production volume [1]:

$$
c_{p t}=a_{p} x_{t-1}^{-b_{p}},
$$

where $a_{p}$ - labor costs of agents for the production of the first product, $b_{p}$ - speed of a product labor intensity reduction with increase in the cumulative production volume.

Let us substitute the expression (6) in the formula (5) and find the labor costs for manufacturing the finished product in $t$ period:

$$
C_{p t}=a_{p} x_{t-1}^{-b_{p}} u_{t}, t=1, T .
$$

The production volumes of the finished product $u_{t}$ are chosen by the principal while planning production activities based on its goal function.

Several options are considered as a goal function of the principal.

1. Minimization of the discounted cumulative labor costs of all agents producing a new product:

$$
J_{p}=\sum_{t=1}^{T} \frac{a_{p} x_{t-1}^{-b_{p}} u_{t}}{\left(1+r_{p}\right)^{t}} \rightarrow \min ,
$$

where $r_{p}$ - discount rate of the principal.

2. Maximization of discounted profit from the production of a new product:

$$
J_{p}=\sum_{t=1}^{T} \frac{P_{t-1} u_{t}-a_{p} x_{t-1}^{-b_{p}} u_{t}}{\left(1+r_{p}\right)^{t}} \rightarrow \text { max, }
$$

where $P_{t-1}$ - price of the new product.

3. Maximization of the production volume of a new product:

$$
J_{p}=x_{T} \rightarrow \max \text {. }
$$

4. Minimization of implementation time of the new product development project:

$$
J_{p}=T \rightarrow \min .
$$

Let us formulate the dynamic task of planning the production volumes of a new product for the principal.

The dynamic planning task consists of finding optimal production volumes $u_{t}^{\text {opt }}, t=1, n$ satisfying the constraint (4), which transfer the dynamic production system (1) from the initial state (2) to the final state (3) and deliver the extremum of one of the goal functions of the principal (8) - (11).

To solve the formulated optimal control problem, Bellman's dynamic programming method [7], implemented in the Free pascal programming environment, was used. Formulation and solutions for dynamic planning problems are given in [8].

As a result of solving the problem of dynamic planning, the principal determines the optimal production volumes of a new product $u_{t}^{o p t}, t=1, n$. To implement the project, it is necessary that the production volumes of the product and parts match. But the choice of the actual production volumes of parts, from which the finished product is assembled, is made by agents upon their own interests. The principal influences the production process through the mechanism of material incentives, encouraging agents economically to fulfill the planned production volumes.

\subsection{The decision-making model of an agent}

The dynamics of the production activity of the $i$-th agent who manufactures the parts for a new product is described by a discrete equation:

$$
y_{t}=y_{t-1}+v_{t}, \quad t=1, T,
$$

where $y_{t}$-cumulative production volume by the agent for the $t$-th time period, $v_{t}$ - production volume by the agent at the period $t$.

The choice of the production volume $v_{t}$ at the period $t$ is the agent's management.

In the initial period, the number of parts produced by agent is known:

$$
y_{0}=Y_{0},
$$

in the final period, the cumulative volume of the parts produced by the agent should be equal to the specified volume:

$$
y_{T}=Y_{0}+R,
$$

where $R$ - specified number of parts, which coincides with the number of finished products.

The production volume of parts in each period $t$ is imposed by the following restrictions:

$$
Q^{\min } \leq v_{t} \leq Q^{\max }, t=1, T,
$$

where $Q_{i}^{\text {min }}-$ minimum parts production volume, considering technological and logistic requirements, $Q^{\text {max }}-$ maximum parts production volume limited by the production capacity of equipment.

Restrictions on the parts production volume coincide with the restriction on the final product production volume. 
The agent labor costs in monetary terms in the period $t$ are defined as the multiplication of part labor intensity $c_{a t}$, the cost of the norm-hour at the enterprise $s$, and the parts production volume in this period $v_{t}$ :

$$
C_{a t}=s c_{a t} v_{t}, t=1, T \text {. }
$$

Due to the learning effect, the labor intensity of parts decreases depending on the cumulative production volume [1]:

$$
c_{a t}=a_{a} y_{t-1}^{-b_{a}},
$$

where $a_{a}$ - agent's labor costs for the first part production, $b_{a}$ - speed of a part labor intensity reduction with increase in the cumulative production volume.

Substituting the expression (17) in the formula (16) the agent's labor costs in the period $t$ can be found:

$$
C_{a t}=s a_{a} y_{t-1}^{-b_{a}} v_{t}, t=1, T \text {. }
$$

Principal uses a dynamic proportional incentive system for the project implementation:

$$
\sigma_{t}\left(\alpha_{t}, v_{t}\right)=\alpha_{t} v_{t}, t=1, T \text {, }
$$

where $\alpha_{t}$-agent's payment rate for a manufactured part in the periods $t=1, T$ (parameters of the incentive function).

Parameters of the incentive function are principal management, through which the principal affects the economic interests of the agent, stimulating him to choose the planned production volumes.

The amount of financial incentives for an agent should not exceed a limited payroll budget $F$ :

$$
\sum_{t=1}^{T} \alpha_{t} v_{t} \leq F, i=1, n
$$

The goal function of the agent is to maximize the discounted income:

$$
J_{a}=\sum_{t=1}^{T} \frac{\sigma_{t}\left(\alpha_{t}, v_{t}\right)-C_{a t}}{\left(1+r_{a}\right)^{t}} \rightarrow \max ,
$$

where $r_{a}$ - agent's discount rate.

The agent income is the difference between the financial incentives and his labor costs, expressed in monetary terms.

Taking into account (18) and (19), the agent goal function (21) will be as follows:

$$
J_{a}=\sum_{t=1}^{T} \frac{\alpha_{t} v_{t}-s a_{a} x_{t-1}^{-b_{a}} v_{t}}{\left(1+r_{a}\right)^{t}} \rightarrow \max
$$

The stated problem is the problem of discrete system optimal control for an agent. The solution of the stated problem is an optimal control $v_{t}^{\text {opt }}, t=1, n$, satisfying constraint (15), which transfers the discrete system (12) from the initial state (13) to the final state (14) and maximizes the agent's discounted income (22). Alongside, the solution of the agent's optimization task depends on the payment rates for the production unit $\alpha_{t}, t=1, n$, which are given by the principal.

To solve the formulated optimal control problem, Bellman's dynamic programming method [7], implemented in the Free pascal programming environment, was used.

\subsection{Algorithm for solving the dynamic incentive problem}

Let us formulate the problem of agent dynamic incentive:

$$
\begin{aligned}
& J_{p}=\sum_{t=1}^{T} g_{p}\left(t, u_{t}, x_{t-1}\right) \rightarrow \max (\min ), \\
& x_{t}=x_{t-1}+u_{t}, t=1, T, \\
& Q^{\min } \leq u_{t} \leq Q^{\max }, \quad t=1, T, \\
& x_{0}=X_{0}, \\
& x_{T}=X_{0}+R, \\
& J_{a}=\sum_{t=1}^{T} \frac{\alpha_{t} v_{t}-s a_{a} y_{t-1}^{-b_{a}} v_{t}}{\left(1+r_{a}\right)^{t}} \rightarrow \max , \\
& \sum_{t=1}^{T} \alpha_{t} v_{t} \leq F, \\
& y_{t}=y_{t-1}+v_{t}, \quad t=1, T, \\
& Q^{\min } \leq v_{t} \leq Q^{\max }, t=1, T, \\
& y_{0}=Y_{0}, \\
& y_{T}=Y_{0}+R .
\end{aligned}
$$

where $g_{p}$ - specific form of the goal function of the principal, is determined by one of the expressions (8) - (11).

The formulated problem (23) represents a dynamic game, the solution of which determines the conditions for coordination between the principal and the agent. The solution of the dynamic game will be the parameters of the incentive system and the production volumes of the parts $\alpha_{t}^{*}, v_{t}^{*}=u_{t}^{*}, t=1, T$, that deliver the extremum to the goal functions of the principal and the agent.

Let us formulate an algorithm for solving the dynamic problem of proportional incentive.

1. The optimal control problem for the principal is solved using Bellman's dynamic programming method, the new product optimal planned volumes $u_{t}, t=1, T$ are found.

2. The principal management is being set - the parameters of the incentive function $\alpha_{t}, t=1, T$ should meet the condition of not exceeding the agent's payroll budget: 


$$
\sum_{t=1}^{T} \alpha_{t} u_{t}\left(\alpha_{t}\right) \leq F
$$

3. For the given parameters of the incentive function $\alpha_{t}, t=1, T$ the optimum control problem for the agent is solved using the Bellman dynamic programming method, and the agent's optimal response is determined as an actual part production volumes $v_{t}\left(\alpha_{t}\right), t=1, T$.

4. The coincidence condition of the planned and actual production volumes that the agent chooses is checked:

$$
\sum_{t=1}^{T}\left(u_{t}-v_{t}\left(\alpha_{t}\right)\right)^{2} \leq \varepsilon
$$

where $\varepsilon$ - predetermined small value. If the condition is satisfied, then the dynamic proportional incentive problem is solved. If not, then the parameters of the incentive function $\alpha_{t}, t=1, T$ are changed and the step 2 is repeated. The principal goal functions (8) - (11) considered above do not depend on the function of financial incentives. Let's formulate the goal functions of the principal, taking into account the expenses of the principal for the agent incentives.

1. Minimization of discounted costs for agent incentives:

$$
J_{p}=\sum_{t=1}^{T} \frac{\alpha_{t} v_{t}}{\left(1+r_{p}\right)^{t}} \rightarrow \min .
$$

2. Maximization of discounted income from the agent's production activity:

$$
J_{p}=\sum_{t=1}^{T} \frac{\left(p_{t}-\alpha_{t}\right) v_{t}}{\left(1+r_{p}\right)^{t}} \rightarrow \max ,
$$

where $p_{t}$ - part price produced by the agent.

In this case, the task of the agent dynamic incentive is as follows:

$$
\begin{aligned}
& J_{p}=\sum_{t=1}^{T} g_{p}\left(t, v_{t}, \alpha_{t}\right) \rightarrow \max (\min ), \\
& J_{a}=\sum_{t=1}^{T} \frac{\alpha_{t} v_{t}-s a_{a} y_{t-1}^{-b_{a}} v_{t}}{\left(1+r_{a}\right)^{t}} \rightarrow \max . \\
& y_{t}=y_{t-1}+v_{t}, t=1, T, \\
& Q^{\min } \leq v_{t} \leq Q^{\max }, t=1, T, \\
& y_{0}=Y_{0}, \\
& y_{T}=Y_{0}+R .
\end{aligned}
$$

where $g_{p}$ - specific form of the goal function of the principal, is determined by one of the expressions (24) - (26).

The solution of the dynamic game will be the parameters of the incentive function and the parts production volumes $\alpha_{t}^{*}, v_{t}^{*}, t=1, T$, that deliver the extremum to the goal functions of the principal and the agent. Let us formulate an algorithm for solving the dynamic problem of proportional incentive in the case when the goal function of the principal depends on the incentive costs (26):

1. The parameters of the principal incentive function $\alpha_{t}, t=1, T$ are given.

2. For the given parameters of the incentive function $\alpha_{t}, t=1, T$ the optimum control problem for the agent is solved using the Bellman dynamic programming method and the agent's optimal response is determined - the actual part production volumes $v_{t}\left(\alpha_{t}\right), t=1, T$.

3. The found agent's response $v_{t}\left(\alpha_{t}\right)$ is substituted into the optimal control problem for the principal, which is solved by Bellman's dynamic programming method. Thus the optimal parameters of the incentive function $\alpha_{t}\left(v_{t}\left(\alpha_{t}\right)\right), t=1, T$ are found.

4. The condition of coincidence of the parameters of the incentive function at the given iteration and the previous one is checked:

$$
\sum_{t=1}^{T}\left(\alpha_{t}\left(v_{t}\left(\alpha_{t}\right)\right)-\alpha_{t}\right)^{2} \leq \varepsilon,
$$

where $\varepsilon$ - predetermined small value. If the condition is satisfied, then the dynamic proportional incentive problem is solved. If not, the parameters of the incentive function must be changed and the step 2 repeated.

\section{Results and Discussion}

The problem of dynamic incentive with the following initial data is considered.

$$
\begin{aligned}
& J_{p}=\sum_{t=1}^{12} \frac{42,64 x_{t-1}^{-0,7} u_{t}}{\left(1+r_{p}\right)^{t}} \rightarrow \min \\
& x_{t}=x_{t-1}+u_{t}, t=1,12 \\
& 0 \leq u_{t} \leq 40, t=1,12 \\
& x_{0}=1, x_{T}=241 \\
& J_{a}=\sum_{t=1}^{T} \frac{\alpha_{t} v_{t}-3837,6 y_{t-1}^{-0,1} v_{t}}{\left(1+r_{a}\right)^{t}} \rightarrow \max \\
& \sum_{t=1}^{T} \alpha_{t} v_{t} \leq 960000 \\
& y_{t}=y_{t-1}+v_{t}, \quad t=1,12 \\
& 0 \leq v_{t} \leq 40, t=1,12 \\
& y_{0}=1, y_{T}=241
\end{aligned}
$$


The numerical solution of the problem was obtained using the proposed algorithm.

The planned trajectory corresponds to the trajectory, which minimizes labor costs of agents and coincides with the rate of a product mastering $b_{p}=-0,7$. At a constant parameters of an incentive function the agent chooses an actual trajectory of the production cumulative volume, which corresponds to an agent's learning rate $b_{a}=-0,1$. Figure 1 shows the planned and actual trajectory of the cumulative volume of production of a new product.

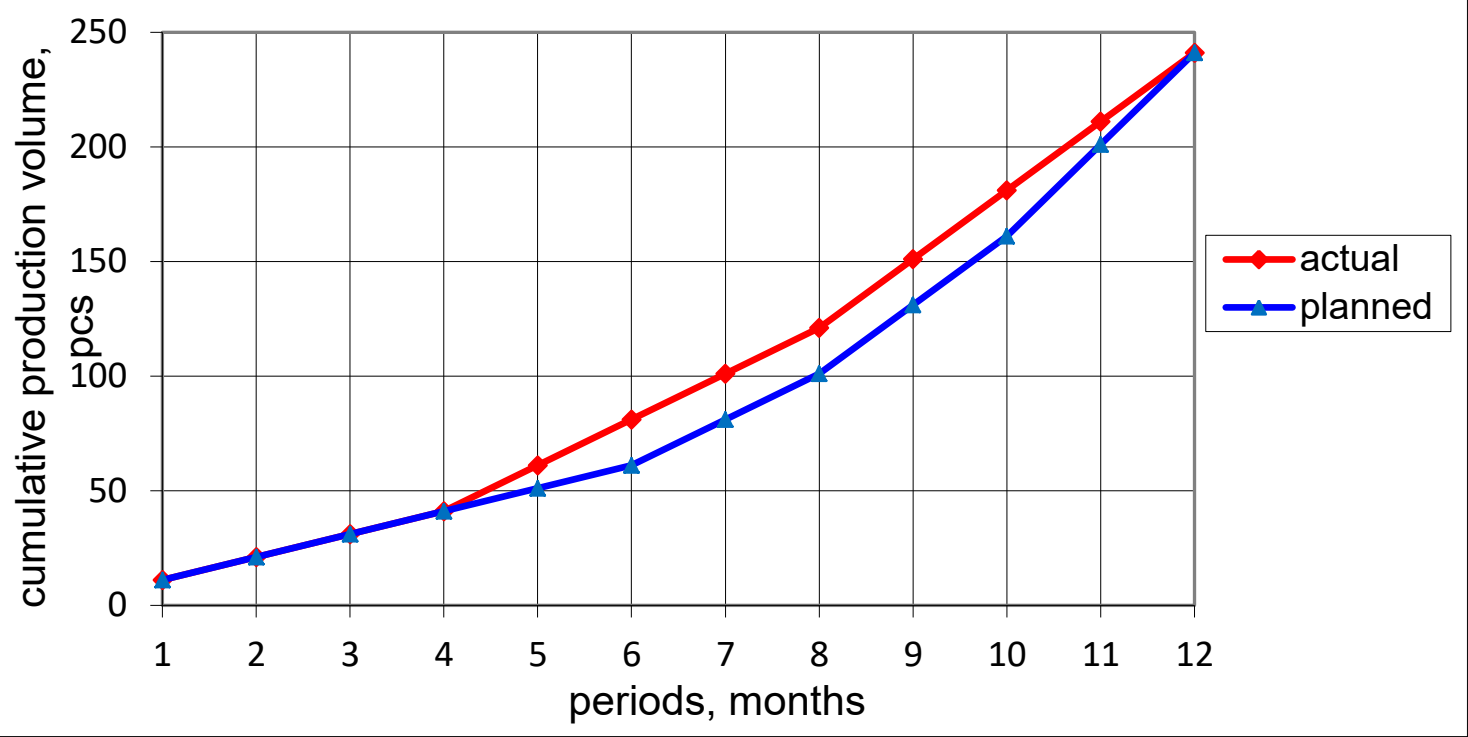

Fig. 1. The planned and actual trajectory of the cumulative volume of production.

In the process of numerical research the following results were obtained:

1. Constant rate of payment does not affect the choice of the agent of the actual cumulative production trajectory. In this case, the trajectory is determined only by the speed of the agent learning.

2. Coordination of the interests of the principal and the agent is achieved using a variable wage rate, which depends on the cumulative volume of production.

3. Applying a payment rate in the form of a linearly increasing function from the cumulative production volume $\alpha_{t}=k y_{t}+d$ makes an agent select the trajectories with a higher learning rate. The agent moves from the "slow" trajectory to the "fast", more "convex" one. The larger value of the control parameter $k$ corresponds to a more "convex" trajectory selected by the agent (Fig. 2).

4. Applying a payment rate in the form of a linearly decreasing function from the cumulative production volume leads to the agent selection of trajectories with a lower learning rate. The agent moves from "fast" trajectory to "slow", less "convex" one. The lager modulo value of the control parameter $k$ corresponds to the agent's chose of a less "convex" trajectory (Fig. 3).

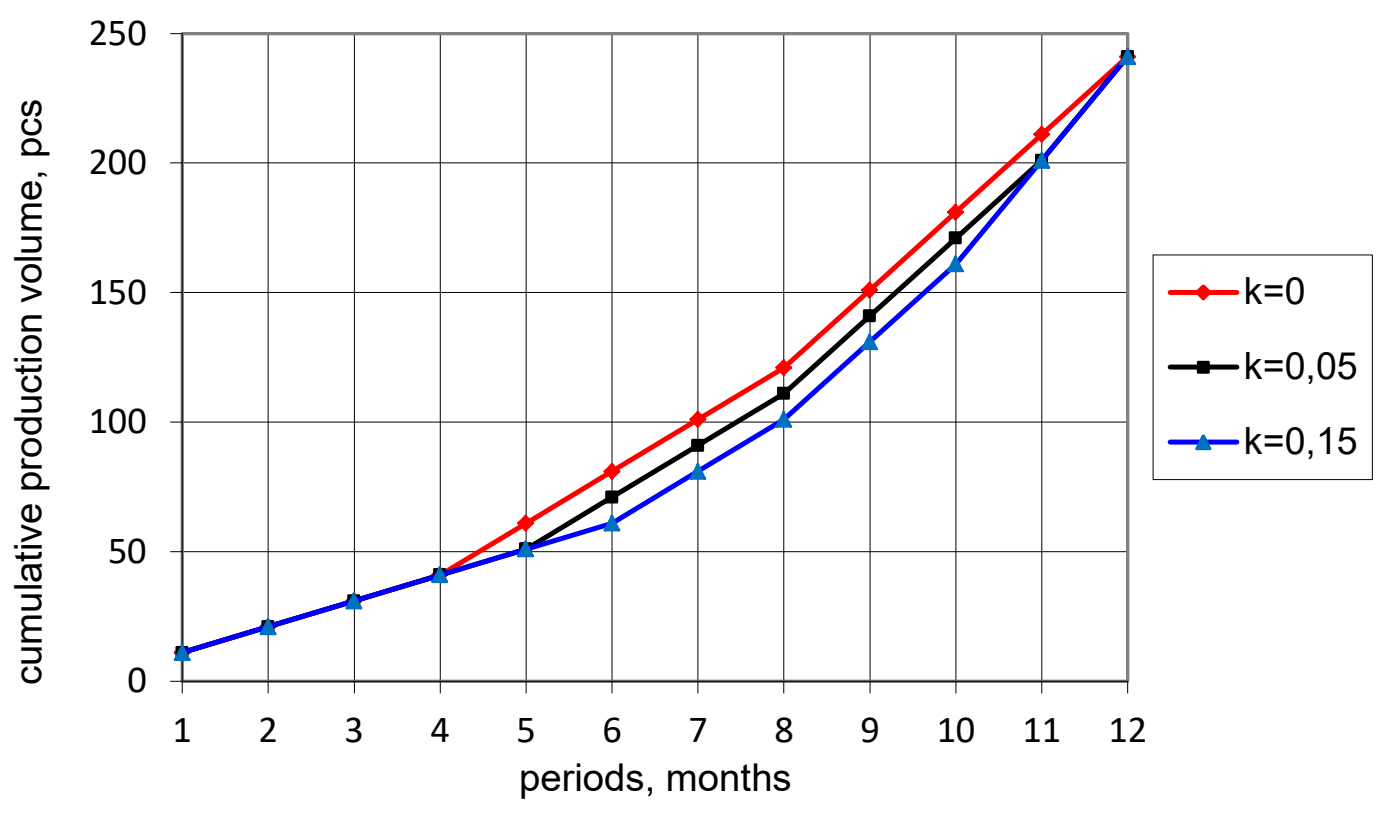

Fig. 2. Influence of parameter $k$ on the actual trajectory of the cumulative volume of production. 


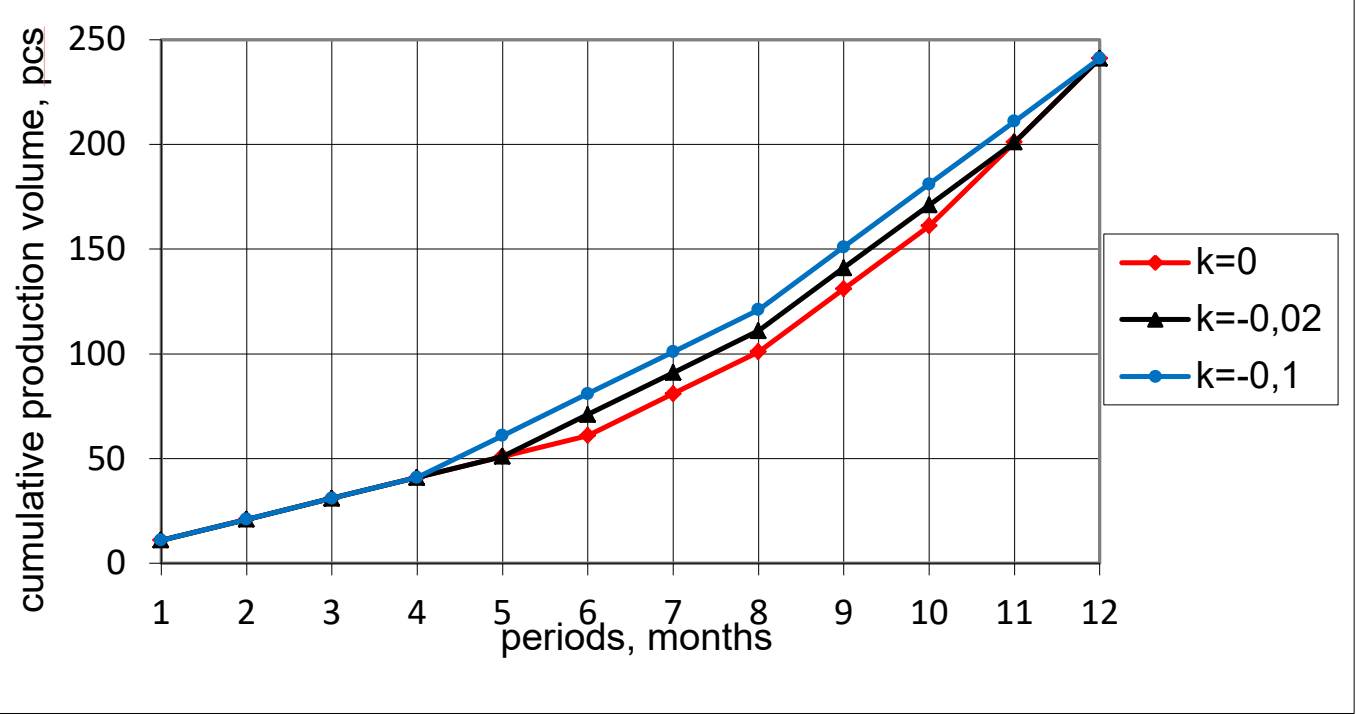

Fig. 3. Influence of parameter $k$ on the actual trajectory of the cumulative volume of production.

\section{Conclusion}

Dynamic decision-making models for the principal and agent in the project for the production of a new product have been developed. The problems of agent dynamic incentive for various goal functions of the principal are formulated. Two options are considered: the goal functions include the costs of material incentives for the agent and do not include them.

For both variants, a numerical algorithm is proposed, based on a sequential solution of two optimal control problems, which are solved using Bellman's dynamic programming method.

A numerical example of the problem solution for the principal goal function, which does not depend on the agent incentive costs, is given. It is shown that the application of the agent salary rate in the form of a linear function, that depends on the cumulative production volume, ensures that the agent selects the planned trajectory of the principal.

\section{Acknowledgements}

The reported study was funded by RFBR and Samara region according to the research project № 17-46-630606.

\section{References}

[1] Wright TP. Factors affecting the cost of airplanes. Journal of the aeronautical sciences 1936; 3(4): $122-128$.

[2] Novikov DA. Models of learning in the work process. Large-scale Systems Control 2007; 19: 5-22.

[3] Gorelik VA, Gorelov MA, Kononenko AF. Analysis of conflict situations in management systems. M.: Radio and communication, 1991.

[4] Basar T, Olsder GJ. Dynamic Noncooperative Game Theory. Philadelphia: SIAM, 1999.

[5] Ougolnitsky GA. Management of sustainable development of active systems. Rostov-on-Don: Publishing of Southern Federal University, 2016 ; 940 p.

[6] Dockner E, Jorgensen S, Long NV, Sorger G. Differential games in economics and management Science. Cambridge: Cambridge University Press, 2000.

[7] Bellman R. Dynamic programming. M.: Foreign Literature Publishing House, 1960.

[8] Pavlov OV. Dynamic optimization of production activities of the enterprise taking into account learning curve effect. Vestnik of Samara State Economics University 2015; 3(125): 88-92. 